\title{
Gedanken zur prognostischen Geographie
}

Vortrag, gehalten vor der Sektion «Geographie und Kartographie» anläßlich der Jahresversammlung der Schweiz. Naturforschenden Gesellschaft in Aarau, 4. Oktober 1975.

«Die Erforschung der Vergangenheit und des Raumes an sich ist leer und enttäuschend, denn die wahre Wissenschaft ist die Wissenschaft der Zukunft, die nach und nach durch das Leben verwirklicht wird."

(Pierre Teilhard de Chardin)

Wir alle setzen uns täglich - bewußt oder unbewußt mit Zukunftsmöglichkeiten und damit mit Prognosen auseinander. Jede Entscheidung, gleichgültig auf welcher Ebene wir sie treffen, ist zukunftsbezogen. Die Fixpunkte für unser Handeln müssen wir nicht hinter, sondern vor uns suchen. Beschlüsse, welche wir heute fassen, Maßnahmen, welche wir heute treffen, beispielsweise in den Bereichen des Umweltschutzes und der Raumplanung, werden sich erst später, in einigen Jahren, z. T. sogar in einigen Jahrzehnten, voll auswirken. Durch die großen Erfindungen und Entwicklungen in Wissenschaft und Technik sind wir immer mehr zum Mitproduzenten unserer Zukunft und auch derjenigen künftiger Generationen geworden. Wohl ist die Zukunft nicht machbar, aber doch zu einem Teil beeinflußbar. Wenn wir aber diese Entwicklungsprozesse beeinflussen wollen, bedingt dies, $\mathrm{da} ß$ wir uns ständig mit den Entwicklungsproblemen, den -perspektiven, den -zielen und den -maßnahmen auseinandersetzen. Eine solche Auseinandersetzung basiert auf theoretischem Wissen, praktischer Erfahrung und intuitiver Begabung: "Wissen ohne Erfahrung und Intuition führt zu wirklichkeitsfremdem Theoretisieren; Erfahrung ohne Wissen und Intuition zu einem hilflosen, nach rückwärts gerichteten Historismus; die Intuition schließlich kann die Seele eines Künstlers zum Ausdruck bringen, vermag aber noch keinen sinnvollen wirtschaftspolitischen Entscheid zu tragen. Anderseits verstärken sich die drei Entscheidungselemente in ihrer Wirkung gegenseitig: Die Kombination von Wissen, Erfahrung und Intuition ist stets mehr als nur die Summe der drei isoliert betrachteten Elemente»l). Es stellt sich nun die Frage, welchen wissenschaftlichen Beitrag die Geographie zur Bewältigung der Zukunft leisten soll und kann.

Die systematische und kritische Behandlung von $\mathrm{Zu}$ kunftsfragen wird als Futurologie bezeichnet. Dabei können drei Aspekte der Futurologie unterschieden werden:

1. Zukunftsforschung i.e.S.: Prognosen usw.

2. Zukunftsgestaltung: Planung usw.

3. Zukunftsphilosophie: Ethik usw.

Auch wenn es bei der Behandlung von Zukunftsfragen in der Praxis oft schwierig ist, diese drei Aspekte scharf voneinander zu trennen, sollen sich die folgenden Ausführungen schwergewichtig auf die Beziehungen der Geographie zur Zukunftsforschung i.e.S. beziehen.

Die Betrachtungsweise in der Geographie ist nicht nur eine räumliche, sondern auch eine zeitliche und somit eine raum-zeitliche. Die zeitliche Dimension spielt in der Geographie eine entscheidende Rolle. Sie tritt uns in zwei Aspekten gegenüber, nämlich in Untersuchungen von «Genesen» und "Geschichten» (z. B. Kulturlandschaftsgenese und Kulturlandschaftsgeschichte): «Man forscht nach der Genese von $\mathrm{Er}$ scheinungen nicht nur in der physischen Geographie, sondern auch in der Kulturgeographie, indem man der Entfaltung und Entwicklung einzelner Elemente, ganzer Ensembles, ganzer ,Landschaften' nachgeht. Und man treibt ,Geschichte der Kulturlandschaft', indem man deren Werden auf einen aus Gründen der Quellenlage oder aus sachlicher Entscheidung gewählten früheren Zustand zurückführt»'2). Auch beim Blick in die Zukunft können wir diese beiden Aspekte unterscheiden, nämlich die Vorausschätzung des künftigen Verlaufes und die Ermittlung künftiger, (zeitlich) fixierter Zustände.

Die Geographen beschäftigten sich aber bisher hauptsächlich mit der Gegenwart und mit der Vergangenheit (Aktualgeographie und historische Geographie). Nur wenige geographische Publikationen setzen sich vorläufig ausschließlich mit der Zukunft auseinander. Auch aktualgeographische Arbeiten behandeln nicht die Gegenwart, sondern die Zeit, welche Haggett sehr treffend als «historical present» oder «recent past» bezeichnet ${ }^{3}$. Grund dafür ist die Zeitspanne, welche zwischen den letzten verfügbaren Statistiken, Luftbildern, Karten usw. und der Veröffentlichung einer aktualgeographischen Untersuchung besteht.

PD Dr. Hans Elsasser, ORL-Institut der ETH, Gebäude Hil, Hönggerberg, 8093 Zürich 
Bis jetzt waren es vor allem die Wirtschaftswissenschafter und die Statistiker, welche prognostische Probleme behandelten ${ }^{4}$. Seit den sechziger Jahren ist allerdings auch die Zahl der Geographen, die sich im Rahmen ihrer wissenschaftlichen Untersuchungen mit Zukunftsproblemen auseinandersetzen, im Steigen begriffen. Dabei bewegen sich diese geographischen Untersuchungen auf zwei verschiedenen Ebenen:

1. In verschiedenen ausländischen und schweizerischen anthropogeographischen Untersuchungen vor allem aus dem Bereich der angewandten Geographie werden auch Aussagen über mögliche künftige Entwicklungen und/oder Zustände des Untersuchungsobjektes gemacht. Bei physischgeographischen Untersuchungen sind zukunftsorientierte Aussagen eher noch selten.

2. Auf einer anderen Ebene liegen diejenigen Arbeiten, in welchen sich Geographen grundsätzlich zur Frage äußern, ob und wenn ja, in welcher Art und Weise sich die Geographen als Wissenschafter mit der Zukunft auseinandersetzen sollen. Nach Haggett lautet die Frage allerdings nicht mehr «Should geographers forecast?», sondern bereits "How should geographers forecast?»5).

Diese Tendenzen berechtigen, von einer prognostischen Geographie als neuen Zweig der Geographie zu sprechen und ihr einen gleichberechtigten Platz neben der historischen Geographie und der Aktualgeographie einzuräumen.

Es darf aber nicht übersehen werden, da $\beta$ es auch heute noch verschiedene Geographen - wie auch andere Wissenschafter - gibt, deren Haltung gegenüber der Futurologie skeptisch bis ablehnend ist. Daraus resultiert dann auch eine negative Einstellung gegenüber der prognostischen Geographie. Diese Einstellung finden wir beispielsweise bei GILDEMEISTER, welcher 1973 schrieb: "Angesichts ihres im Grunde genommen nicht wissenschaftlichen Charakters kann die Prognose daher nicht Bestandteil irgendeiner Wissenschaft sein, somit auch nicht Bestandteil der Geographie»6). Die Geographie sollte sich somit auf die Erforschung von vergangenen und gegenwärtigen Ereignissen, welche geschehen und unveränderbar sind, beschränken und sich nicht mit Zukunftsmöglichkeiten beschäftigen. Nur gesicherte Ereignisse können Bestandteil der geographischen Forschung sein. Im
Gegensatz zur Vergangenheit und Gegenwart gibt es ja nicht eine einzige Zukunft, sondern verschiedene Zukunftsmöglichkeiten.

Diametral entgegengesetzt argumentieren andere Forscher, welche behaupten, daß eine Disziplin erst dann zur Wissenschaft werde, wenn sie auf ihrem Gebiet den Blick in die Zukunft geöffnet habe, oder zu öffnen verspricht; oder anders ausgedrückt, da $\beta$ das Endziel jeder wissenschaftlichen Tätigkeit die Prognose sei: «The essence of science is prediction»7). Zum wesentlichen Problem der Wissenschaftlichkeit von Voraussagen schreibt GERFIN: «Trotz aller Mängel und Schwächen, die den Prognoseansätzen heute noch anhaften und zum Teil vermutlich immer anhaften werden, besteht kein Grund, über alle Bemühungen der Vorausschätzung den Bann zu sprechen. Wissenschaftliche Prophetie freilich ist unmöglich und der Versuch dazu ... unmoralisch. Auf zahlreichen, wenn auch nicht auf allen Gebieten können dennoch wissenschaftlich fundierte Urteile über die zukünftige Entwicklung getroffen werden, denen Seriosität nicht abzusprechen ist und die für zukunftsorientierte Entscheidungen eine verläßlichere Basis schaffen als die reine Intuition, sofern ihre Ergebnisse nicht apodiktisch interpretiert werden. Die wissenschaftliche Fundierung liegt darin, da $\beta$ sich die Aussagen auf den jüngsten Stand der theoretischen Forschung stützen und daß alle relevanten Informationen, mit adäquaten analytischen Werkzeugen aufbereitet, einbezogen werden. $\mathrm{Da}$ aber die Wissenschaft nur aufzeigen kann, ob eine Theorie in sich widerspruchsfrei und zudem in der Lage ist, Vergangenheit und Gegenwart hinreichend zu erklären, bleibt der letzte, wichtige Schritt, die Abschätzung der Wirklichkeitsnähe der aufgestellten Beziehungen für die Zukunft, ein Akt des - außerwissenschaftlichen - Urteils. ${ }^{8)}$

Welches sind nun die speziellen Argumente für die prognostische Geographie? Diese können folgendermaßen zusammengefaßt werden:

1. Zweck der Prognose ist, die augenblicklich bestehenden Unsicherheiten über die Zukunft zu reduzieren. «Ausmaß und Zeitpunkt dieser Reduzierung machen die Nützlichkeit der Prognose aus.»19) Es wäre aber ein fatales Mißverständnis annehmen zu wollen, Prognosen könnten je die Unsicherheiten über die Zukunft vollständig eliminieren. Man würde damit eine Prophezeiung verlangen. Die Prognose sagt nicht 
«So wird es sein», sondern "so könnte es (unter gewissen Annahmen) sein». Auch die Geographie sollte deshalb in Zusammenarbeit mit andern Wissenschaften einen Beitrag zu dieser Reduzierung von Unsicherheiten leisten, um dadurch dem handelnden Menschen ein richtiges Disponieren zu erleichtern.

2. Die Raumprognose bildet ein wichtiges Bindeglied zwischen der Raumanalyse und der Raumdiagnose einerseits sowie der Raumplanung und Raumgestaltung anderseits. Dies ist deutlich ersichtlich bei LOWRY ${ }^{10)}$, welcher drei Stufen von Raummodellen unterscheidet: "descriptive models», "predictive models» und "planning models». Wenn die Geographie nicht nur die heutigen räumlichen Strukturen - unter Berücksichtigung der bisherigen Entwicklung - beschreiben und kausal erklären, sondern vermehrt als bisher einen Beitrag zur Planung und Gestaltung unseres Lebensraumes leisten will, muß sie den Schritt zur Raumprognose wagen, "so ungewohnt und unsicher eine derartige Aufgabe und die exakte Formulierung der Ergebnisse auch erscheinen mag»11).

3. Die Beschäftigung mit der Zukunft schärft aber auch unsern Blick für die Probleme und Möglichkeiten der Gegenwart: «Our results are intended to show possibilities and problems; they are not forecasts»12). Einem solchen Einsatz der Prognose in der Geographie dürfte auch von Gegnern einer prognostischen Geographie kaum Widerstand erwachsen, wird doch hier die Prognose «lediglich» als Instrument der Erforschung der aktuellen Raumsituation eingesetzt. Gleichzeitig wird aber auch deutlich, daß unsere Gegenwart und unser gegenwärtiges Entscheiden und Handeln nicht nur durch die Vergangenheit, sondern auch durch die Zukunft bestimmt wird. In diesem Zusammenhang muß darauf hingewiesen werden, da $\beta$ viele Prognosemethoden nicht nur zeitlich vorwärts-, sondern auch rückwärtsgerichtet angewandt werden können, beispielsweise wenn fehlende historische Informationen rekonstruiert werden müssen.

4. Eine Verlängerung der zeitlichen Betrachtungsweise in der Geographie in Richtung Zukunft bedeutet keineswegs eine Abkehr vom bisherigen geographischen Forschungsweg, sondern eine Erweiterung und Bereicherung der geographischen Forschung. Diese Verlängerung darf aber niemals dazu führen, daß darob die Analyse und Diagnose der aktualgeographischen Situation vernachlässigt wird. Grundlage jeder Pro- gnose ist die gründliche, kausale Analyse und Diagnose: «Forecasting is simply good analysis» ${ }^{13)}$.

Geographische Prognosen sind in die Zukunft gerichtete geographische Forschungen. Somit können alle Erscheinungen und Prozesse, um deren Beschreibung und Erklärung sich die Geographie bemüht, Gegenstand von Prognosen sein. Das Gebiet der geographischen Voraussage ist deshalb ebenso umfassend wie das Forschungsfeld der Geographie. Es ist folglich nicht möglich, über die (geographische) Prognose schlechthin zu diskutieren. Prognosen können - entsprechend ihrem jeweiligen Zweck - nach verschiedenen Gesichtspunkten gegliedert werden:

\section{Blickrichtung14):}

In der Praxis müssen Prognosen zwei wichtige, unterschiedliche Aufgaben erfüllen:

a) Aufzeigen der künftigen Entwicklung und Zustände aufgrund bestehender Entwicklungstendenzen = explorative Prognosen, z. B. Status-quo-Prognosen;

b) Aufzeigen von zielgerichteten Entwicklungen auf bestimmte, gewünschte Zustände hin = normative Prognosen, Zielprognosen.

Der Vergleich zwischen einer erwarteten und der zielgerechten Entwicklung, d.h. zwischen der explorativen und normativen Prognose bildet ein wesentliches Element in jedem Planungsprozeß. Dabei ist es aber unbedingt notwendig, daß nicht nur die Endzustände, sondern auch die Zwischenetappen und der Weg, welcher von der heutigen Situation zum gewünschten, bzw. zum erwarteten Endzustand führt, aufgezeigt werden. Diese Konfrontation erlaubt dann, die Maßnahmen(bündel) zu entwickeln, welche notwendig sind, um die Entwicklung vom Trend weg in Richtung Erfüllung der Zielvorstellungen zu steuern. In der Praxis zeigt sich allerdings, daß sich die wesentlichen methodischen Unterschiede zwischen beiden Prognosearten beim Einsatz ihrer Ergebnisse nicht immer einhalten lassen. Annahmen in der Status-quo-Prognose enthalten häufig Zielelemente, während Zielprognosen in der Regel nicht unbeeinflußt von Entwicklungstrends festgelegt werden ${ }^{15}$.

Der Geograph wird sich im Rahmen seiner Arbeiten vermutlich in erster Linie mit explorativen Prognosen und innerhalb dieser vor allem mit Status-quo-Prognosen beschäftigen. Wenn er aber raumbedeutsame Pro- 
zesse nicht nur beschreiben, erklären und voraussagen, sondern auch lenken will, ist es notwendig, daß er sich zusätzlich mit den Problemen der normativen Prognosen auseinandersetzt. "Unsere Wissenschaft ist aus einer Wissenschaft der Zustände zu einer Wissenschaft der Veränderungen geworden, und sie wird zu einer Wissenschaft der Lenkungen werden.»16)

\section{Total-(Global-) und Partialprognosen:}

Totalprognosen versuchen die Gesamtentwicklung eines Raumes vorauszusagen. Partialprognosen betreffen dagegen nur einzelne Elemente, d. h. Geofaktoren. Zwischen Global- und Partialprognosen bestehen enge, wechselseitige Zusammenhänge. Sehr häufig wird man eine Totalprognose benötigen, um die gewünschte Partialprognose zu erstellen. Anderseits bedarf es wiederum Voraussagen über die Entwicklung der wichtigsten Geofaktoren, um die Gesamtentwicklung eines Raumes voraussagen zu können.

Das Schwergewicht prognostischer Tätigkeit in der Geographie sollte mehr bei Total-, denn bei Partialprognosen liegen. Zusammenhänge zwischen den Geofaktoren zu ermitteln und zu erklären, darf als eine zentrale Aufgabe der Geographie bezeichnet werden. Sehr oft sind Voraussagen über die Entwicklung einzelner Geofaktoren weniger wichtig, als Prognosen über die Zusammenhänge der Geofaktoren: «Prognosen von Relationen sind wichtiger als Prognosen von Daten $)^{17)}$.

\section{Objekt, Aggregationsgrad:}

Partialprognosen können nach den zu prognostizierenden Objekten gegliedert werden. Eine Gegenüberstellung einzelner Prognosearten und der entsprechenden Teildisziplinen der Geographie ergibt folgendes Bild:

$\begin{array}{ll}\text { Bevölkerungsprognose } & \text { Bevölkerungsgeographie } \\ \text { Arbeitsplatzprognose } & \text { Wirtschaftsgeographie } \\ \text { Wohnbauprognose } & \text { Siedlungs- und } \\ & \text { Stadtgeographie } \\ \text { Verkehrsprognose } & \text { Verkehrsgeographie } \\ \text { usw. } & \text { usw. }\end{array}$

Diese Aufstellung zeigt, daß in sehr vielen Prognosebereichen Einsatżmöglichkeiten für den Geographen bestehen. Jeder Geograph besitzt ja neben seinen allgemeinen geographischen Kenntnissen auch vertiefte, spezialisierte Kenntnisse in einer oder mehreren Teildisziplinen und ihren Nebenfächern.

Bei der Unterteilung in Total- und Partialprognosen sowie bei der weiteren Unterteilung der Partialprognosen spielt der Aggregationsgrad eine wichtige Rolle. «Welcher Aggregationsgrad für eine bestimmte Prognose sinnvoll und nützlich ist, hängt von dem vorliegenden Problem, d. h. der Art der gesuchten Variablen und ihrer Stellung im ökonomischen System sowie von dem Verwendungszweck der Untersuchungsergebnisse $a b .{ }^{18)}$

\section{Zeitdauer:}

Allgemein eingebürgert hat sich eine Dreiteilung der Zukunft in eine nahe, mittlere und ferne Zukunft, bzw. der Prognosen in kurz-, mittel- und langfristige Prognosen. Die Spanne, welche diese Zeiträume umfaßt, ist allerdings von Autor zu Autor recht unterschiedlich, wie das die folgenden Beispiele zeigen:

$\begin{array}{llll}\text { Prognosen } & \text { Graf }{ }^{19)} & \begin{array}{l}\text { Phlipponneau } \\ \text { 20) }\end{array} & \text { Kühnn21) } \\ & \text { Jahre } & \text { Jahre } & \text { Jahre } \\ \text { kurzfristige } & \text { bis } 1 & 4-5 & 3-5 \\ \text { mittelfristige } & 1-3 & 15 & 10-50 \\ \text { langfristige } & 10 \text { u. m. } & 30 \text { u. m. } & 60 \text { u. m. }\end{array}$

Das Schwergewicht von geographischen Prognosen dürfte in jenem Zeitabschnitt liegen, den ein großer, wenn nicht sogar der größte Teil der heute lebenden Menschen noch erleben wird, d. h. im Bereich der je nach Autor - mittleren oder fernen Zukunft. Konjunkturelle Aspekte bleiben vermutlich in der prognostischen Geographie eher ausgeklammert. Die Länge des sinnvollen Prognosezeitraumes muß im konkreten Fall durch Kausaluntersuchungen abgeklärt werden. Prognosen sind in irgendeinem Zeitpunkt überholt. Unvorhersehbare Ereignisse und Einflüsse können den Verlauf einer vorauszuplanenden und -berechnenden Entwicklung jederzeit verändern. Prognosen müssen deshalb periodisch nachgeführt und korrigiert werden.

\section{Prognoseraum:}

Die Prognosemethode ist abhängig von der Größe des Prognoseraumes. Nicht alle Methoden sind für jede räumliche Einheit geeignet. Dabei gilt: Je größer die 
räumliche Bezugseinheit und je weniger Beziehungen nach außen, desto einfacher die Methode und desto weniger unsicher die Ergebnisse. Das ergibt sich einerseits daraus, daß bei einer großräumigen Betrachtungsweise viele kleinräumlich wirkenden Bestimmungsfaktoren ausgeklammert werden können. Ferner ist zu beachten, daß für kleine Raumeinheiten in der Regel weniger einschlägiges statistisches Material zur Verfügung steht als für große. "Als Faustregel gilt, daß einer regionalen Prognose immer eine Prognose des ihr übergeordneten Raumes vorgeschaltet werden sollte. Gut ist es auch, wenn Prognosen der benachbarten Regionen parallelgeschaltet werden können, nicht nur zur Erfassung der gegenseitigen Einflüsse auf Bevölkerung und Wirtschaft, sondern auch zur Durchführung sogenannter Konsistenztests: Die Summe der Teilprognosen sollte mit der Gesamtprognose übereinstimmen.»22)

\section{Quantitative und qualitative Prognosen:}

Quantitative Prognosen sind auf Zahlenmaterial aufgebaut und geben eher mengenmäßige Aussichten an. Die qualitativen Voraussagen befassen sich entweder mit der Qualität des Untersuchungsobjektes und seinen Verbesserungsmöglichkeiten oder umschreiben zukünftige Entwicklungen nur verbal. Quantitative Methoden spielen bei der Erstellung von Prognosen eine bedeutende Rolle. Der quantitativen Geographie kommt deshalb innerhalb der prognostischen Geographie eine wichtige Stellung zu: «Models are to use, not to believe»23).

In diesem Zusammenhang muß noch auf die Genauigkeit von Prognosen hingewiesen werden. Maßgebend für die Anforderungen an die Genauigkeit sind die Zwecke der Prognose. Für viele Zwecke genügt schon die Bestimmung der Entwicklungsrichtung oder von ungefähren Angaben. «Jede Prognose ist von Natur aus ungenau.»24) «Stimmen Vorausschätzung und Wirklichkeit trotzdem überein, ist dies in der Regel mehr dem Zufall als den Fähigkeiten des Bearbeiters zu verdanken.»25) Durch den Einsatz von geeigneten Methoden muß man versuchen, die Risikomarge zu verringern. Es ist unzweckmäßig, Genauigkeitsgrade anzustreben, die gar nicht gefragt sind. «Die Prognosemethoden sollten jedoch auf das jeweils schwächste Glied im vorhandenen Unterlagenmaterial und benötigten Instrumentarium ausgerichtet sein; es hat kei- nen Sinn, einen Klotz teils mit dem Beil und teils mit Filigrantechnik zu bearbeiten. ${ }^{26)}$ Dabei gilt es stets zu berücksichtigen, daß die geforderte Genauigkeit oder Zuverlässigkeit der Vorausschätzung und der Aufwand für eine Prognose in einem vernünftigen Verhältnis zueinander stehen sollen.

Die Wahl der geeignetsten Prognosemethoden und Prognosetechniken hängt nun in entscheidendem $\mathrm{Maße}$ von diesen Punkten oder Vorfragen (Blickrichtung, Objekt, Zeitdauer, Prognoseraum, Genauigkeit) ab. Wir müssen uns damit abfinden, daß es keine einheitliche und optimale Methode für alle Fälle gibt. Es soll an dieser Stelle auf eine Darstellung der verschiedenen Prognosemethoden und -techniken verzichtet und auf die entsprechende Spezialliteratur verwiesen werden, insbesondere auf die Zusammenstellung von $\mathrm{JANTSCH}^{27)}$, welche über hundert Methoden und Techniken enthält. Es ist nun eine Aufgabe der prognostischen Geographie, diese Methoden im Rahmen von geographischen Untersuchungen anzuwenden, zu testen sowie nötigenfalls zu ergänzen und zu verbessern.

Zum Abschluß muß noch auf den didaktischen Wert der Beschäftigung mit Zukunftsproblemen hingewiesen werden: Im Bericht "Umweltforschung in der Schweiz»28) wird folgende Frage'aufgeworfen: "Wie kann und soll bei der Bevölkerung das Verständnis für die Inhalte der Zukunftsforschung gefördert werden, bzw. wie kann die Bevölkerung aus der engen örtlichen und zeitlichen Betrachtungsweise losgelöst werden?» Eine Antwort darauf gibt GARRISON in seinem Aufsatz "Future Geographies»: «Society needs creative action by creative individuals. What better stage could there be for the education of creative people than the context of future geographies and the challenge to create improved geographies.1 ${ }^{29)}$ 


\section{Anmerkungen}

1) KNESCHAUREK, F., Entwicklungsperspektiven und Probleme der schweizerischen Volkswirtschaft, Bankverein-Heft Nr. 9 (1975), S. 8 f.

2) PFEIFER, G., Vergangenheit, Gegenwart und Zukunft - Zeit und Raum in der Geographie, in: Geographische Zeitschrift, Heft 33 (1973), Festschrift für Prof. Dr. Ernst Plewe, S. 15

3) Vgl. dazu HAGGETT, P., Forecasting alternative spatial, ecological and regional futures: problems and possibilities, in: CHORLEY, R. J. (Hg.), Directions in Geography, London 1973, S. 221

4) Eine Zusammenstellung von internationalen und nationalen Stellen mit statistischem und prognostischem Tätigkeitsbereich findet sich bei ABT, R., Langfristige Prognosen im europäischen Raum, in ORL-DISP Nr. 31 (1973), S. 10

5) HAGgETT, P., Geography: A modern Synthesis, New York 1972, S. 441

6) GILDEMEISTER, R., Landesplanung, Das geographische Seminar, Braunschweig 1973, S. 48

7) Nach GERFIN, H., Langfristige Wirtschaftsprognose, Tübingen und Zürich 1964, S. 8 ff.

8) GERFIN, H., a. a. O., S. $194 \mathrm{f}$.

9) MÜLLER, J. H., Methoden zur regionalen Analyse und Prognose, Taschenbücher zur Raumplanung Bd. 1, Hannover 1973, S. 104

10) Vgl. dazu LOWRY, I. S., A short course in model design, in: Journal of the American Institute of Planners Nr. 2, 1965, S. 159

11) WITT, w., Planerische Utopie und geographische Realität, in: Mitteilungen der Österreichischen Geographischen Gesellschaft, Band 114/I/II/1972, S. 25

12) STONE, R. U. a., Economic Growth and Manpower, Bacie Spring Conference 1963, London 1964, S. 13, zit. nach GERFIN, H., a. a. O., S. 194, Fußnote 10

13) BASSIE, V. L., Economic Forecasting, New York, Toronto and London 1958, Preface, S. VI, zit. nach GERFIN, H., a. a. O., S. 2

14) Vgl. dazu RossI, A., Prognostik und Raumplanung, in: Grenzen der Raumplanung, ORL-Institut ETH, Zürich 1975 (im Druck)
15) Nach DIETRICHS, B., Staus-quo-Prognosen und Zielprojektionen im Raumplanungsproze $B$ - Funktionen und Konflikte, in: Informationen zur Raumentwicklung, Heft 4/5, Regionalisierte Zielprojektionen für Bevölkerung und Arbeitsplätze, 1975, S. $137 \mathrm{ff}$.

16) STRZYGOWSKI, W., Diskussionsbemerkung auf dem Geographentag in Bad Godesberg 1967, zit. nach wITT, w., a. a. O., S. 25

17) MAURER, J., Methodik der Raumplanung I, ORLSchriftenreihe Nr. 14, ORL-Institut ETH, Zürich 1973, S. 83

18) GERFIN, H., a. a. O., S. 22

19) St. Galler Zentrum für Zukunftsforschung (H.GRAF), Seminar «Wirtschaftsprognostik», Kursunterlagen, HSG, St. Gallen 1973

20) Phlipponneau, M. (Hg.), Géographie et perspectives à long terme, UGI Commission de Géographie appliquée IVe Symposium Rennes, 15.-22. Juli 1971, Sablé 1973, S. 459

21) KÜHN,A., Mittlere Zukunft, in: Handwörterbuch der Raumforschung und Raumordnung, herausg. von der Akademie für Raumforschung und Landesplanung, Bd. II, Spalte 1992 ff., Hannover 1970

22) NYDEGGER, A., Grundlagen und Methoden für Infrastrukturprognosen, in: Infrastruktur, ORL-Schriftenreihe Nr.3, ORL-Institut ETH, Zürich 1969, S. 16

23) HAGGETT, P., Forecasting ..., a. a. O., S. 234

24) NYDEGGER, A., a. a. O., S. 14

25) SCHWARZ,K., Methoden der Bevölkerungsvorausschätzung unter Berücksichtigung regionaler $\mathrm{Ge}-$ sichtspunkte, Taschenbücher zur Raumplanung, Bd. 3, Hannover 1975, S. 2

26) NYDEGGER, A., a. a. O., S. 15

27) JANTSCH, E., Technological Forecasting in Perspective, OECD, Paris 1967

28) Eidg. Amt für Umweltschutz und Amt für Wissenschaft und Forschung, Umweltforschung in der Schweiz, Bd. 3: Fragenkatalog, Bern 1975, S. 63

29) GARRISON, W. L., Future Geographies, in: CHORLEY, R. J. (Hg.), Directions in Geography, London 1973, S. 248 


\section{Zusätzliche, in den Anmerkungen nicht enthaltene Literatur}

CHISHOLM, M., (Ltg.), Regional forecasting, Colston Papers No. 22, London 1970

GEHMACHER, E., Methoden der Prognostik. Eine Einführung in die Probleme der Zukunftsforschung und Langfristplanung, rombach hochschul paperback, bd. 29, Freiburg i. Br. 1971

JOUVENEL, B. DE, Die Kunst der Vorausschau, Neuwied und Berlin 1967

METRON, Zur Methodik der Bevölkerungsprognose, Brugg 1970 (Vervielfältigung)

PICHT, G., Prognose, Utopie, Planung - Die Situation des Menschen in der Zukunft der technischen Welt, Schriften der Vereinigung Deutscher Wissenschaftler, Heft 6, Stuttgart 1968
ROTHSCHILD, K. W., Wirtschaftsprognose. Methoden und Probleme, Heidelberger Taschenbücher, Bd. 62, Berlin u. a. 1969

SCHMID, B., Bevölkerungsprognose, Migration und Markovketten, ORL-Bericht Nr.33, ORL-Institut ETH, Zürich 1975

SCHMID, B., Entropie-Modelle und Raumplanung. Herleitung und Interpretation, ORL-Bericht, ORL-Institut ETH, Zürich 1975 (im Druck)

SCHUMACHER, G., Planung, Bevölkerungsentwicklung und Prognose, in: Beiträge zur heutigen Humangeographie (Festschrift Winkler), Geographisches Institut ETH, Publ. Nr. 55, Zürich 1975

THEIL, H., Economic Forecasts and Policy, Contributions to Economic Analysis, Bd.15, 2. überarbeitete Auflage, Amsterdam 1961 